Commun. math. Phys. 5, 97-105 (1967)

\title{
Space-Time and Degrees of Freedom of the Elementary Particle*
}

\author{
H. BACRY** \\ Argonne National Laboratory, Argonne, Illinois \\ and The Institute for Advanced Study, Princeton, New Jersey
}

Received February 25, 1967

\begin{abstract}
First, a general property of Lie groups is used in the case of the Poincaré group in order to define the one particle phase space. It is eight-dimensional in the general case and six-dimensional for a spinless or massless particle.

Embedding the Poincaré group into the similitude group of space-time permits us to interpret the dilatation operator as a dynamical variable. The connection between the similitude group and field equations is discussed.

LurÇAT's ideas on a possible dynamical role of spin and mass-spin spectra of particles (Regge trajectories) are discussed under the point of view of the degrees of freedom.
\end{abstract}

\section{Introduction}

When one compares the usual description of a free particle in classical and quantum mechanical cases, one is faced with two very different approaches: on the one hand, one starts from position and velocity variables and all observables are functions of these variables; on the other hand, the aspect is group theoretical since a particle is associated with an irreducible projective representation of the space time group $G$ (Galileo or Poincaré group). Spin is ignored in the first case but naturally involved in the second one by group theoretical arguments. Several models ${ }^{1}$ have been built for the description of a classical spinning particle generally in increasing the number of degrees of freedom, attributing in this way to the particle an internal structure. In our opinion, it is essential to limit the number of new parameters in order that the particle posseses all the characteristics of an elementary particle. The usual definition of the particle concept in quantum mechanics suggests that we search for a group theoretical definition of the classical (spinning) particle based on

* This work constitutes a completed version of a preprint entitled "Classical Hamiltonian Formalism for Spin", Argonne, September, 1966.

** On leave from Université de Marseille, France. Work supported in part by the National Science Foundation.

1 A very large number of articles have been devoted to this subject and it seems not necessary to give here a complete list of references. Nevertheless, we will mention the Thomas model $[1,2,7]$ in which no "internal structure" is involved. 
the properties of the Poincaré group. Such a definition is given in section II. Covariance requirements and field equations are shown to need an enlarged phase space. Possible new degrees of freedom are obtained in section III in embedding the Poincaré group into larger groups. Some consequences are investigated in connection with LURÇAT's ideas on a possible dynamical role of spin and fields describing sets of particles on Regge trajectories. Finally, in an appendix, we give the way of defining phase spaces from any Lie group.

\section{The One Particle Phase Space}

Invariance under the group $G$ is a characteristic of every free system and it is known that in a Lagrangian formulation, this invariance property furnishes - through the Noether theorem - conservation laws, one law for each one parameter subgroup of $G$. This is true in both mechanics. For instance, a free relativistic spinless particle of mass $m$ is usually described by the classical Lagrangian

$$
\mathscr{L}=m \sqrt{\dot{x}^{2}}
$$

where $x$ describes the four coordinates of a point on the wordline followed by the particle and the dot means a derivation with respect to an arbitrary time parameter. The invariance of $\mathscr{L}$ under the Poincaré group implies the conservation of the energy-momentum vector

$$
p=\frac{\partial \mathscr{L}}{\partial \dot{x}}=\frac{m \dot{x}}{\sqrt{\dot{x}^{2}}}
$$

and that of the generalized angular momentum

$$
M=x \wedge p .
$$

One could hope to find a Lagrangian for a spinning particle which would furnish the equation $\dot{\mathrm{s}}=0$ where $\mathrm{s}$ is the spin vector. Because we required the elementary character of the particle, it is necessary that the only Lagrange equations would be $\dot{p}=0$ and $\dot{\mathrm{s}}=0$. It seems impossible to find a Lagrangian satisfying those conditions. In the same way, the Lagrangian (II.I) is obviously unable to describe the motion of a massless particle. These two difficulties lead us to discard any Lagrangian formalism and start from the following postulate:

Postulate 1. To each generator $A_{\alpha}$ of the Poincaré group is associated in a one-to-one correspondence a conserved quantity: it is a self-adjoint operator $\mathscr{A}_{\alpha}$ on the Hilbert space in quantum mechanics and a real parameter $a_{\alpha}$ in classical mechanics.

The role of this postulate consists in replacing the effects of the Noether theorem on a Lagrangian formalism. It associates to every free system a set of ten conserved quantities without distinguishing between 
composite and elementary systems (see postulate 2 ). To each quantum mechanical observable built in terms of the $\mathscr{A}_{\alpha}$ 's corresponds a classical observable as it is required by the Bohr correspondence principle but this mapping is not one to one.

Since we are interested in problems involving one particle, we must restrict the operators $\mathscr{A}_{\alpha}$ to the Hilbert subspace associated with the given particle. Two of the observables, namely the mass $m$ and the spin $s$ are fixed (i.e., they are multiples of the identity operator). This remark induces the following definition of the concept of a classical elementary particle.

Postulate 2. The classical observables of a free particle are functions on the manifold $\mathscr{M}$ defined in the ten-dimensional space of the $a_{\alpha}$ 's by the two following equations

$$
\begin{aligned}
m^{2}\left(a_{\alpha}\right)=m^{2} & \text { (constant) } \\
\mathbf{s}^{2}\left(a_{\alpha}\right)=s^{2} & \text { (constant). }
\end{aligned}
$$

The manifold $\mathscr{M}$ is the phase space associated to the particle of mass $m$ and spin s.

The manifold $\mathscr{M}$ is obviously eight-dimensional in the general case where $m^{2}>0$ and $s^{2}>0$. It becomes six-dimensional when $\mathrm{s}^{2}$ becomes zero. More precisely, if the ten variables $a_{\alpha}$ are written in the form $p_{0}, \mathbf{p}, \mathbf{j}, \mathbf{k}$ (respectively associated with the time translation, space translation, rotation and pure Lorentz transformation generators), the mass $m$ and the spin $\mathrm{s}$ are defined by the well-known relations ${ }^{2,3}$

$$
\begin{gathered}
m^{2}=p_{0}^{2}-\mathbf{p}^{2} \\
m \mathbf{s}=p_{0} \mathbf{j}+\mathbf{p} \times \mathbf{k}-\frac{\mathbf{j} \cdot \mathbf{p}}{m+p_{0}} \mathbf{p} .
\end{gathered}
$$

In the case of massless particles, the spin must be replaced by the helicity $s$ defined as the ratio of the classical Thomas-Pauli-Lubanski polarization vector $w$ and the energy momentum

$$
w_{\mu}=s p_{\mu} .
$$

It is easy to prove in this last case that the phase space is also sixdimensional.

2 The vector $\mathbf{s}$ and the classical counterpart of the Pauli-Lubanski vector $w$ have been first mentioned by L. H. Thomas $[1,2]$. See for instance H. BacRY $[7,8]$.

${ }^{3}$ Note that Eq. (II.7) expressing $\mathbf{s}$ in terms of $p_{0}, \mathbf{p}, \mathbf{j}$ and $\mathbf{k}$ is the same as that which expresses the transformation property of the magnetic field $(\mathbf{j}$ and $-\mathbf{k}$ are respectively replaced by the magnetic and the electric fields at rest, $\mathbf{s}$ by the magnetic field relative to an observer with speed $\left.\mathbf{p} / p_{0}\right)$. The analogy with the quantum mechanical case is given in Ref. [9-10]. 
The fact that all these manifolds can be interpreted as phase spaces is known in group theory (see appendix). In order to make it evident, we must give a set of canonical coordinates on it. If we define the vector

the eight variables

$$
m \mathbf{x}=\mathbf{k}-\frac{\mathbf{p} \times \mathbf{j}}{m+p_{0}}-\frac{\mathbf{k} \cdot \mathbf{p}}{p_{0}\left(m+p_{0}\right)} \mathbf{p}
$$

where

$$
\begin{aligned}
& x^{\alpha}=(x, y, z, \varphi) \\
& p_{\alpha}=\left(p_{x}, p_{y}, p_{z}, s_{z}\right)
\end{aligned}
$$

$$
\varphi=\arccos \frac{s_{x}}{\sqrt{s^{2}-s_{z}^{2}}}
$$

provide a set of conjugate variables for the eight dimensional phase space. To prove it, one defines the Poisson bracket of two observables $f$ and $g$ as follows

$$
\{f, g\}=\frac{\partial f}{\partial x^{\alpha}} \frac{\partial g}{\partial p_{\alpha}}-\frac{\partial g}{\partial x^{\alpha}} \frac{\partial f}{\partial p_{\alpha}}
$$

To each observable $f$ can be associated a differential operator $f$ acting on the space of observables in the following way

$$
\left\{\begin{array}{l}
f\left(x^{\alpha}, p_{\alpha}\right) \rightarrow \hat{f} \\
\hat{f}(g)=\{f, g\} .
\end{array}\right.
$$

In particular, one has the properties

$$
\left\{\begin{array}{l}
\left\{x^{\alpha}, p_{\beta}\right\}=\delta_{\beta}^{\alpha} \\
\left\{x^{\alpha}, x^{\beta}\right\}=0 \\
\left\{p_{\alpha}, p_{\beta}\right\}=0
\end{array}\right.
$$

It is easy to verify that the Hamiltonian ${ }^{4}$

$$
H=\sqrt{\mathbf{p}^{2}+m^{2}}
$$

leads us to the usual equations of motion and to the conservation of $\mathbf{p}, \mathbf{j}$, $k$ and $\mathbf{s}$.

The cases of a spinless (resp. massless) particle is readily obtained by dropping the variables $s_{z}$ and $\varphi$ (resp. $s_{z}, \varphi$, and $m$ ).

With our definition of phase space, the problem of quantizing the free elementary particle consists only in replacing the variables $a_{\alpha}$ by Hermitian operators; consequently we are led to the search for a Hermitian irreducible representation of the Lie algebra of the Poincare group ${ }^{5}$.

${ }^{4}$ Note that for a non-relativistic spinning particle in an external field $(A, V)$, the motion is described by the Hamiltonian

$$
H=\frac{(\mathbf{p}-e \mathbf{A})^{2}}{2 m}+e V-g \frac{e}{2 m} \mathbf{s} \cdot \mathbf{B}
$$

where $\mathbf{B}=\operatorname{rot} \mathbf{A}$ (see Kramers, Ref. [3]).

5 These phase spaces have been recently quantized by SourIaU (see Ref. [11]). 


\section{Going Farther}

Can we enlarge the phase space in order to include mass or spin or both as dynamical variables? Such a question is suggested on one hand by the covariance requirement (the four components of the energymomentum vector must play analogous roles as they do in the Lagrangian formalism (II.1): the constancy of $p^{2}$ is a consequence of the Lagrange equations) and on the other hand by the possibility of interactions where particles are "off mass shell". It could be also useful, following LuRÇAT's ideas $[13,14]$ to attribute a dynamical role to the spin variable $s$, permitting it to go "off spin shell" when interactions are involved.

A natural way for enlarging the phase space consists in embedding the Poincaré group in a larger group, for instance in considering the similitude group of space time $S$ instead of the Poincare group. In this particular case, the spin is still an invariant of the group and there is no other invariant. The group $S$ has eleven generators, those of the Poincaré group and the dilatation generator $D$. The commutation relations involving $D$ are ${ }^{6}$

$$
\begin{aligned}
{\left[D, P_{0}\right] } & =i P_{0} \\
{[D, \mathbf{P}] } & =i \mathbf{P} \\
{[D, \mathbf{J}] } & =0 \\
{[D, \mathbf{K}] } & =0 .
\end{aligned}
$$

Following the way followed in the above section, one defines the ten dimensional manifold - the enlarged phase space - generated by the eleven parameters $p_{0}, \mathbf{p}, \mathbf{j}, \mathbf{k}$ and $d$ (observable associated with $D$ ) restricted by the condition $\mathbf{s}^{2}=$ constant. The even dimensionality property is, of course, essential to interpret the manifold as a phase space. We must find a set of canonical variables. Such a set is given by

$$
\begin{aligned}
& \xi^{\mu}=(\boldsymbol{\xi}=m \mathbf{x}, \varphi, d) \\
& \pi_{\mu}=\left(\boldsymbol{\pi}=\mathbf{p} / m, s_{z}, \log m\right)
\end{aligned}
$$

as it can be easily verified using generalized Poisson brackets. In fact, according to the preceding footnote ${ }^{6}$, one verifies that $\{d, \xi\}=\{d, \pi\}=0$. Because $\varphi$ and $s_{z}$ are dimensionless, $\{d, \varphi\}=\left\{d, s_{z}\right\}=0$. On the other hand, one has

and

$$
\{d, m\}=m
$$

$$
\{d, f(m)\}=m f^{\prime}(m) \text {. }
$$

We must choose the function $f(m)$ in such a way that $m f^{\prime}(m)=1$. We are readily led to the solution $f(m)=\log m$.

\footnotetext{
${ }^{6}$ If $\mathscr{A}$ is an observable with the dimension of length ${ }^{n}$, one has $[D, \mathscr{A}]=-$ in $\mathscr{A}$.
} 
Eq. (II.16) becomes in terms of the new variables

$$
H=e^{\log m} \sqrt{\boldsymbol{\pi}^{2}+1}
$$

and the Hamilton equations

$$
\left\{\begin{array}{l}
\frac{d \log m}{d t}=-\frac{\partial H}{\partial d}=0 \\
\frac{d d}{d t}=\frac{\partial H}{\partial(\log m)}=H
\end{array}\right.
$$

are easily integrated. They give us

$$
\left\{\begin{array}{l}
m=\text { constant } \\
d=\int H d t
\end{array}\right.
$$

and provide us a relation between the action and the dilatation generator.

It is interesting to mention that, for the spinless particle phase space, one can find a covariant set of canonical coordinates, namely the $p_{\mu}$ 's, the four components of the energy-momentum vector and their four conjugate variables $x^{\mu}$ defined as follows

$$
x^{\mu}=\frac{M^{\mu v} p_{\nu}+d p^{\mu}}{p_{\lambda} p^{\lambda}} .
$$

(It is easy to check the well-known relations $\left\{x^{\mu}, x^{\nu}\right\}=0,\left\{p_{\mu}, p_{\nu}\right\}=0$ and $\left\{x^{\mu}, p_{\nu}\right\}=-\delta_{\nu}^{\mu}$.)

The interest of the above remark is its relation with the KleinGordon equation relative to a spinless particle. In the field equation one uses functions on a four dimensional space (four degrees of freedom) but the equation selects an irreducible spinless representation of the Poincaré group. In other words

4 degrees of freedom -1 equation

$$
=3 \text { degrees of freedom (spinless particle) }
$$

One can try to make a new step in embedding the group $S$ into a larger group for which the spin is no longer an invariant. The lowest step consists in adding one degree of freedom, providing us a 12-dimensional phase space. One of the possibilities is the phase space associated to the conformal group on space time, a 15 dimensional Lie group; the most general phase space is 12 dimensional since this group has three fundamental invariants $(15-3=12)$. By associating a field equation to this group, we may write a relation analogous to (III.9), namely

6 degrees of freedom -1 equation $=5$ degrees of freedom .

Therefore, such a model describes a spectrum of particles, that is to say a set of particles with a relation between mass and spin. Such models have already been proposed by physicists [15-21].

As another example, one can choose a 12 dimensional phase space associated to the direct product $S^{\prime} \times S L(2, C)$ where $S^{\prime}$ is a group iso- 
morphic to $S$ and describes the orbital part of motion. The phase subspace associated to $S^{\prime}$ is therefore eight dimensional. That of $S L(2, C)$ is four dimensional since $S L(2, C)$ has six generators and two invariants. A field equation which corresponds to $S^{\prime} \times S L(2, C)$ is the generalized Dirac equation

$$
\left(i \gamma^{\mu} \partial_{\mu}+m_{0}\right) \psi=0
$$

where the $\gamma^{\mu}$ 's are not subjected to any condition. Such a field equation describes also a mass-spin spectrum of particles [15-21] and all operators are in fact built with the 12 operators $x^{\mu}, p_{\mu}, \gamma^{\mu 7}$. The connection between phase space and quantum mechanics is certainly less evident in that case since the field has discrete components but our arithmetic proves that it would be possible to write equations for fields which are functions on a 6-dimensional manifold, the direct product of the Minkowski space, on which acts as usual the group $S^{\prime}$, and of the complex line (a two dimensional real space) on which acts the group $S L(2, C)$ in the following way. defines

Let $\Lambda=\left|\begin{array}{ll}a & b \\ c & d\end{array}\right|$ be a matrix of $S L(2, C)$ and $z$ a complex number. One

$$
\Lambda z=\frac{a z+b}{c z+d} .
$$

An analogous model has been recently discussed in Ref. [22]. One of the consequences of the above discussion is that LURÇAT's hypothesis of a dynamical role of spin implies the following properties (mass and spin are supposed to be different from zero for the sake of simplicity).

1. The homogeneous space on which the fields are defined is at least six dimensional.

2. In the case of a six-dimensional homogeneous space, a field associated with one irreducible representation of the Poincaré group obeys two equations corresponding to the two invariants of the group. If only one equation is required, the field under consideration describes a mass-spin spectrum of particles.

3. For a larger homogeneous space, as that investigated by LURÇAT [13-14], it is necessary to interpret the new degrees of freedom which are not required by a dynamical role of spin alone.

Apart from this problem, it would be interesting to examine in our scheme the role of the dynamical groups in connection with the number of degrees of freedom. We hope to come back to this question in the future.

${ }_{7}$ The usual Dirac equation describes only one spin because the $\gamma^{\mu}$ 's are satisfying the relation $\gamma^{\mu} \gamma^{\nu}+\gamma^{\nu} \gamma^{\mu}=2 g^{\mu \nu}$. 
Acknowledgements. The author is grateful to Dr. H. Ekstein for stimulating discussions and to Professor R. HAAG for his critical comments. Thanks are due to Dr. Peshioin for hospitality extended to him at the Physics Division, Argonne National Laboratory and to Professor J. RoBert OpPenHeImer for the hospitality of The Institute for Advanced Study.

\section{Appendix}

In this appendix, we give the general proof of the possibility of defining, in a canonical way, phase spaces from any Lie group. I am grateful to Professors B. Kostant and J. M. Souriau for imparting to me that proof.

Let $A$ be a Lie algebra of a Lie group $G$ and $A^{*}$ the dual vector space of $A$. We will denote the action of an element $f$ of the dual on an element $x$ of $A$ by

$$
\langle f, x\rangle \text {. }
$$

The adjoint representation is defined as follows

$$
x, y \in A, \quad x \rightarrow \operatorname{ad}(x) \quad \operatorname{ad}(x) y=[x, y] .
$$

The co-adjoint representation (the contragredient representation of the adjoint representation) is given by

$$
\begin{gathered}
x, y \in A, f \in A^{*}, \quad x \rightarrow \operatorname{coad}(x) \\
\langle\operatorname{coad}(x) f, y\rangle=\langle f,[y, x]\rangle=-\langle f, \operatorname{ad}(x) y\rangle .
\end{gathered}
$$

Let $f$ be an element of $A^{*}$ and $A_{f}$ the subalgebra of $A$ which leaves $f$ invariant $\left(A_{f}\right.$ is the isotropy subalgebra of the element $\left.f\right)$. The elements of $A / A_{f}$ generate the tangent space of the orbit $O_{f}$ at the point $f$. Eq. (A.3) induces on this tangent space a symplectic structure described by

$$
X, Y \in A / A_{f} \quad(X, Y)_{f}=-(Y, X)_{f}=\langle f,[X, Y]\rangle .
$$

The antisymmetric bilinear form (A.4) is defined everywhere on the orbit $O_{f}$ and provides it with a symplectic structure. This property induces the definition of Poisson brackets on $O_{f}$ and permitted us to interpret all orbits in $A^{*}$ as phase spaces for classical mechanics. [In the case of the Poincare group, each orbit is characterized by the value of mass and spin and the tangent vector space $A / A_{f}$ is eight-dimensional in the general case, six-dimensional for spinless or physical massless cases.]

Remarks. 1. Quantization methods can be used in order to find irreducible representations of the Poincaré group. More generally, Kostant [23] proposed to use these methods in order to find all unitary irreducible representations of Lie groups.

2. All symplectic spaces are even dimensional. It would be interesting to understand the connection between this property and the following 
one:

$$
(\mathrm{Obs})=\frac{(\operatorname{dim})+(\mathrm{inv})}{2}
$$

where (dim) is the dimensionality of the Lie algebra, (inv) the number of its fundamental invariants and (obs) the number of generators of a maximal abelian subalgebra of the enveloping Lie algebra. This theorem was implicitly proved by $\mathrm{RACAH}$ [24] for all semi-simple groups, but it seems that the proof could be generalized to a larger class of Lie groups. In fact, the Galileo, the Poincaré, the similitude groups and the inhomogeneous $S L(n, C)$ groups [25] satisfy all this property.

\section{References}

[1] Thomas, L. H.: Nature 117, 514 (1926).

[2] - Phil. Mag. 3, 1 (1927).

[3] Kramers, H. A.: Quantentheorie der Elektron und der Strahlung, sect. 57. Leipzig: Akademische Verlag 1938.

[4] - Physica 1, 825 (1934).

[5] MöLler, C.: Saertryk of Matematisk Tiddskrift B (1950).

[6] Bargmann, V., L. Michel, and V. L. Telegdi: Phys. Rev. Letters 2, 435 (1959).

[7] BACRY, H.: Nuovo Cimento 26, 1164 (1962).

[8] - Ann. de Physique 8, 197 (1963).

[9] - Leçons sur la théorie des groupes et les symétries des particules élémentaires. New York: Gordon and Breach (to appear).

[10] - Lectures on dynamical groups and relativity, Summer Institute for Theoretical Physics, Boulder 1966. New York: Gordon and Breach 1967.

[11] Souriau, J.-M.: Ann. Inst. H. Poincaré (to appear).

[12] - Compt. Rend. Acad. Sci. 263, 1191 (1966).

[13] Lurçat: F.: Physics 1, 95 (1964).

[14] —, and NGHIEM XUAN HaI (in preparation).

[15] Majorana, E.: Nuovo Cimento 9, 335 (1932).

[16] Harish-Chandra: Proc. Roy. Soc. A 192, 195 (1948).

[17] Gelfand, I. M., and A. M. YAGLOM: ZETF 18, 70 (1948).

[18] LÉvy-Leblond, J.-M. : Nuovo Cimento 45, 772 (1966).

[19] Feldman, G., and P. T. Matrhews: Multi-mass fields. Preprint ICTP 66/17.

[20] NaMbU, Y.: Relativistic wave equation for particles with internal structure and mass spectrum (preprint 1966).

[21] Fronsdal, C.: Infinite multiplets and local fields (preprint 1966).

[22] BACRY, H., and J. Nuyts: Mass-spin relation in a Lagrangian model. Phys. Rev. (to appear).

[23] Kostant, B.: U.S.-Japan Conference in Differential Geometry. Kyoto 1965.

[24] RACAH, G.: Group theory and spectroscopy. Lectures given at The Institute for Advanced Study, p. 52, Princeton (1951).

[25] BaCry, H., and A. Kinlberg: Commun. Math. Phys. 1, 150 (1965) 